\title{
Dinâmica inflacionária brasileira: resultados de auto-regressão quantílica*
}

\author{
André Luis Santiago Maia ${ }^{\dagger}$, Francisco Cribari-Neto ${ }^{\ddagger}$
}

Sumário: 1. Introdução; 2 . Os dados; 3. Auto-regressão quantílica; 4. Testes de raiz unitária; 5 . A dinâmica inflacionária brasileira; 6. Conclusão.

Palavras-chave: dinâmica inflacionária brasileira; estacionariedade; raiz unitária; regressão quantílica.

Neste artigo nós estudamos a dinâmica inflacionária brasileira após a implementação do Plano Real em 1994. Nós usamos modelos auto-regressivos quantílicos e testes de raiz unitária baseados em representações autoregressivas quantílicas para caracterizar tal dinâmica. $O$ artigo mostra que a dinâmica inflacionária não apresenta comportamento uniforme ao longo dos diferentes quantis condicionais. Em particular, os resultados fornecem evidência de dinâmica globalmente estacionária, mesmo com o processo alcançando não-estacionariedade na cauda superior da distribuição condicional.

The object of study of this paper is the Brazilian inflationary dynamics after the implementation of the Real Plan in 1994. We use quantile autoregressive models and unit root tests derived from quantile autoregressive representations to characterize such dynamics. It is shown that the inflationary dynamics is not uniform across different conditional quantiles. In particular, the overall dynamics is stationary, even though the time series behavior of the process at the upper tail of the conditional distribution proves to be far from stationary.

Códigos JEL: C14; C22; C13.

\section{INTRODUÇÃO}

Ao longo das últimas décadas, muitos países, tanto desenvolvidos quanto em desenvolvimento, atravessaram processos inflacionários de diferentes magnitudes e duração. Diversos países em desenvolvimento, em especial, enfrentaram hiperinflações ou inflações acentuadas por prolongados períodos

\footnotetext{
* Os autores agradecem comentários e sugestões de dois paraceristas anônonimos e também o suporte financeiro da CAPES e do CNPq.

$\dagger$ Departamento de Estatística, Universidade Federal de Pernambuco, Recife/PE, 50740-540. Email: andremaia@ufpe.br

${ }_{\ddagger}^{\ddagger}$ Departamento de Estatística, Universidade Federal de Pernambuco, Recife/PE, 50740-540. Email: cribari@ufpe.br
} 
de tempo. No caso do Brasil, particularmente, a economia apresentou elevadas e crescentes taxas de inflação por prolongados períodos de tempo, atribuídas ao alto grau de indexação e à existência de inércia inflacionária. Neste contexto, surgiram no Brasil diversas estratégias na tentativa de reduzir e estabilizar estas taxas de inflação. Algumas dessas estratégias foram de natureza mais ortodoxa e outras, de natureza mais heterodoxa. A partir de 1986, foram implementados vários planos de estabilização da inflação brasileira, quase todos de natureza heterodoxa, sendo que a maioria não obteve o sucesso desejado, alcançando apenas alívio imediato e não conseguindo reduzir a inflação de forma sustentada. A estabilização da inflação brasileira só foi conseguida após várias tentativas. Somente o Plano Real, implementado em 1994, através de um sofisticado processo de substituição da moeda, conseguiu eliminar o mecanismo de inflação alta presente na economia brasileira de maneira duradoura. Nesse sentido, ele tem sido um plano de estabilização bem sucedido, tendo como conseqüência uma mudança estrutural na dinâmica inflacionária, que adquiriu caráter quase estacionário (Cassiano, 2003). Embora ainda existam problemas a serem resolvidos, como a questão fiscal, eles não parecem colocar em risco a estabilidade conquistada.

O comportamento da inflação brasileira foi estudado por vários autores. Novaes (1993) realizou um estudo utilizando dados mensais para o período de janeiro de 1970 a dezembro de 1985 e estimou o grau de inércia na inflação brasileira em 1/3. Durevall (1998), para dados de 1968 a 1985, usando uma formulação de correção de erros, estimou o nível de inflação inercial no Brasil em 0.41. Cati et alii (1999), com dados da taxa de inflação mensal que se estendem de janeiro de 1974 a junho de 1993, encontraram que a dinâmica inflacionária brasileira é quase inteiramente inercial, 0.97. Em contraste, a partir da mesma série usada por Cati et alii (1999), Campêlo e Cribari-Neto (2003) encontraram que a componente inercial na dinâmica inflacionária brasileria é de segunda ordem, sendo, assim, negligível.

Os impactos de planos de estabilização na inflação do Brasil têm sido amplamente discutidos em trabalhos empíricos e teóricos. Do ponto de vista da política econômica, é importante identificar estes impactos, bem como os efeitos produzidos por tais planos sobre a dinâmica inflacionária brasileira. Neste contexto, há um interesse particular no exame do comportamento da inflação no Brasil após a implementação do Plano Real, dadas evidências empíricas de que o efeito produzido por este plano de estabilização na dinâmica inflacionária brasileira tem sido positivo. Com relação à inércia inflacionária, após o Plano Real ela parece ter de fato sofrido uma mudança estrutural. Antes de julho de 1994, acreditava-se que a inércia inflacionária era alta e que a inflação havia tornado-se explosiva. A hipótese de inflação inercial afirmava que, devido aos mecanismos de indexação de preços e salários presentes na economia brasileira, choques de oferta eram automaticamente transferidos para a taxa de inflação, criando assim um mecanismo de inércia inflacionária.

No presente artigo, nós estudamos a dinâmica inflacionária brasileira pós-Plano Real através de uma abordagem clássica de modelagem de séries temporais. Mais precisamente, usamos uma classe de modelos auto-regressivos quantílicos, conhecidos como modelos QAR, proposta por Koenker e Xiao (2004a), com a finalidade de fornecer uma alternativa para o estudo de dinâmicas assimétricas e de persistência nas de taxas de inflação brasileiras. Os modelos QAR vêm sendo bastante utilizados em estudos de séries temporais econômicas e podem ser facilmente estimados usando o enfoque de regressão quantílica proposto por Koenker e Bassett Jr (1978). A vantagem destes métodos é que, em vez de confiar exclusivamente em uma única medida de tendência central, eles permitem a análise de quantis condicionais, permitindo, assim, a análise de toda a distribuição condicional da variável resposta. A motivação para o uso dos modelos QAR reside na caracterização da dinâmica inflacionária em diferentes quantis da distribuição condicional da taxa de inflação, permitindo uma investigação mais abrangente da existência de raiz unitária. Para investigar o comportamento inercial da taxa de inflação brasileira, nós usamos, no presente artigo, testes de raiz unitária baseados no enfoque de auto-regressão quantílica.

Cumpre notar que a literatura internacional tem considerado a possibilidade de comportamentos assimétricos de dinâmicas inflacionárias. Por exemplo, Ball et alii (1992) desevolvem um modelo de custos de menu onde choques que aumentam os preços desejados das firmas conduzem a maiores respostas de ajustes de preços do que choques que reduzem preços desejados. Wallis (1999) considera 
previsões da taxa de inflação num contexto de densidades assimétricas de previsão. No presente artigo, nós consideramos o caráter assimétrico da dinâmica inflacionária a partir de uma perspectiva distinta, a saber: a de respostas a choques econômicos. Os resultados obtidos sugerem que há evidência de tal comportamento assimétrico na inflação brasileira no período pós-Plano Real.

\section{OS DADOS}

Foram usados dois indicadores da inflação brasileira. O primeiro foi o Índice Geral de Preços - Disponibilidade Interna (IGP-DI). O IGP-DI é calculado pelo Instituto Brasileiro de Economia (IBRE) da Fundação Getúlio Vargas e tem por finalidade mensurar a variação dos preços, do primeiro ao último dia do mês, que afetam diretamente unidades econômicas situadas no território brasileiro. Este Índice é uma média ponderada de outros três indicadores: Índice de Preços no Atacado (IPA), que mede a variação de preços no mercado atacadista e recebe peso 60\%; Índice de Preços ao Consumidor (IPC), que mede a variação de preços com base em famílias com renda mensal de 1 a 33 salários mínimos nas cidades de São Paulo e Rio de Janeiro e entra com peso 30\%; Índice Nacional da Construção Civil (INCC), que mede a variação de preços no setor da construção civil, considerando tanto materiais como também a mão-de-obra empregada no setor, recebendo peso $10 \%$. A segunda série analisada neste artigo é a da taxa de inflação medida pelo Índice Nacional de Preços ao Consumidor Amplo (IPCA) calculado pelo Instituto Brasileiro de Geografia e Estatística (IBGE). O IPCA reflete a variação dos preços das cestas de consumo das famílias com rendimento mensal de 1 a 40 salários mínimos.

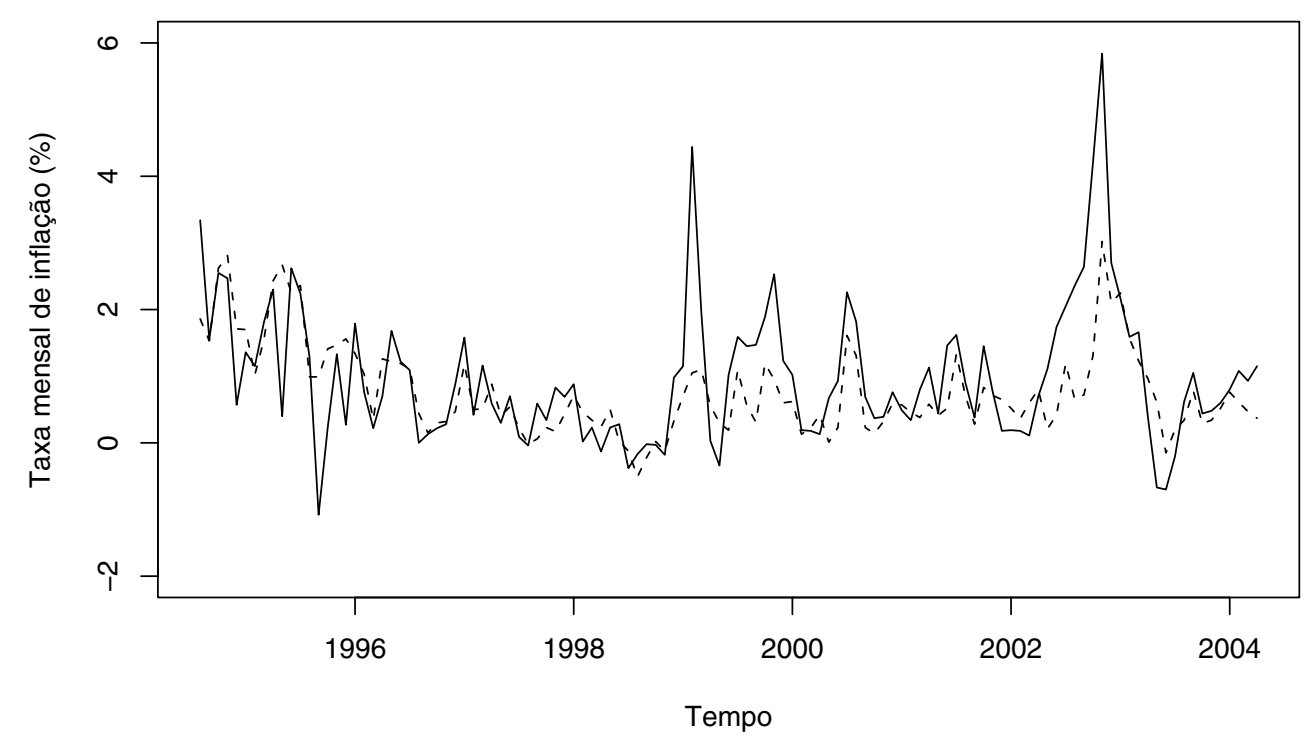

Figura 1 - Taxas mensais de inflação medidas pelo IGP-DI (linha sólida) e IPCA (linha tracejada).

Os dados são mensais, cobrem o período que se estende de agosto de 1994 a abril de 2004 e foram obtidos de http://www. ipeadata.gov.br. As trajetórias das séries do IGP-DI e do IPCA nesses 117 meses estão apresentadas na Figura 1. 


\section{AUTO-REGRESSÃO QUANTÍLICA}

Modelos de séries temporais de coeficientes constantes vêm sendo usados com sucesso em muitas aplicações estatísticas, porém várias especifições de modelos de coeficientes aleatórios cada vez mais vêm sendo utilizados nestas aplicações. Um caso particular desta última classe de modelos é o modelo de séries temporais baseado em regressão quantílica linear. Os modelos de regressão quantílica linear têm recebido considerável atenção, pois conduzem a uma análise estatística mais completa da relação estocástica entre variáveis aleatórias. Entretanto os trabalhos de séries temporais baseados nestes modelos, por exemplo, Knight (1989), Weiss (1991) e Rogers (2001), tendem a tratar do caso em que as inovações (erros) são independentes e identicamente distribuídas (i.i.d.). Esta restrição faz com que os coeficientes auto-regressivos sejam independentes dos quantis especificados. Koenker e Xiao (2004a) consideraram modelos auto-regressivos quantílicos lineares em que os parâmetros auto-regressivos variam com o quantil $\tau \in(0,1)$ na tentativa de obter uma outra opção de modelagem de séries temporais econômicas que apresentam dinâmica assimétrica ou persistência local.

Existe uma literatura incipiente sobre o modelo de auto-regressão quantílica linear, como, por exemplo, Hasan e Koenker (1997) e Koenker e Xiao (2004a). Neste modelo, o $\tau$-ésimo quantil condicional da variável resposta $y_{t}$ é expresso como uma função linear dos valores passados da resposta. Um aspecto fundamental da literatura que trata de modelos auto-regressivos quantílicos é o fato de que o foco reside quase exclusivamente no caso de inovações i.i.d.. Neste caso, as variáveis explicativas exercem o papel clássico de deslocar a posição da densidade condicional de $y_{t}$, mas não afetam a escala ou a forma da densidade.

Discutiremos, aqui, a classe mais geral de modelos auto-regressivos quantílicos, em que todos os coeficientes auto-regressivos são admitidos ser $\tau$-dependentes e onde, diferentemente do caso de inovações i.i.d., as inovações são capazes de alterar não apenas a locação, mas também a escala e a forma da densidade condicional de $y_{t}$. O modelo pode ser definido como a seguir. Seja $\left\{U_{t}\right\}$ uma seqüência de variáveis aleatórias i.i.d. com distribuição uniforme padrão e considere o processo auto-regressivo de ordem $p, A R(p), y_{t}=\alpha_{0}\left(U_{t}\right)+\alpha_{1}\left(U_{t}\right) y_{t-1}+\cdots+\alpha_{p}\left(U_{t}\right) y_{t-p}$, onde os $\alpha$ 's são funções $[0,1] \rightarrow \mathbb{R}$ desconhecidas a serem estimadas. Assim, segue que a $\tau$-ésima função quantil condicional de $y_{t}$ pode ser escrita como

$$
Q_{y_{t}}\left(\tau \mid y_{t-1}, \ldots, y_{t-p}\right)=\alpha_{0}(\tau)+\alpha_{1}(\tau) y_{t-1}+\cdots+\alpha_{p}(\tau) y_{t-p},
$$

ou, de maneira mais compacta, como $Q_{y_{t}}\left(\tau \mid y_{t-1}, \ldots, y_{t-p}\right)=x_{t}^{\top} \alpha(\tau)$, onde $x_{t}=\left(1, y_{t-1}, \ldots, y_{t-p}\right)^{\top}$ e $\alpha(\tau)=\left(\alpha_{0}(\tau), \ldots, \alpha_{p}(\tau)\right)^{\top}$. Aqui, a $\tau$-ésima função quantil condicional da resposta $y_{t}$ é expressa como uma função linear dos valores passados desta variável. Neste modelo, os coeficientes autoregressivos podem ser $\tau$-dependentes e assim variar com os quantis. O caso particular mais simples do modelo de auto-regressão quantílica é sua versão de primeira ordem,

$$
Q A R(1): Q_{y_{t}}\left(\tau \mid y_{t-1}, \ldots, y_{t-p}\right)=\alpha_{0}(\tau)+\alpha_{1}(\tau) y_{t-1}
$$

Diferentemente da maioria dos modelos auto-regressivos de coeficientes aleatórios, em que os coeficientes são assumidos estocasticamente independentes uns dos outros, o modelo $Q A R(p)$ possui coeficientes que são funcionalmente dependentes. Este fato deve-se à exigência de monotonicidade das funções quantis, que impõe alguma disciplina nas formas assumidas pelas funções $\alpha$. Esta disciplina requer essencialmente que o vetor $\alpha(\tau)$, ou alguma transformação sua, seja monótono em cada coordenada. O interesse de muitos pesquisadores recai na utilização do modelo $Q A R(p)$ para verificar o comportamento de raiz unitária em séries temporais econômicas.

De maneira análoga à regressão quantílica, a estimação dos parâmetros do modelo auto-regressivo quantílico linear envolve a resolução do problema

$$
\min _{\alpha \in \mathbb{R}^{p+1}} \sum_{t=1}^{T} \rho_{\tau}\left(y_{t}-x_{t}^{\top} \alpha\right),
$$


onde a função "check" $\rho_{\tau}$ é definida por

$$
\rho_{\tau}(u)=\left\{\begin{array}{l}
\tau u, \quad u \geq 0, \\
(\tau-1) u, \quad u<0 .
\end{array}\right.
$$

A solução para um dado $\tau$, $\widehat{\alpha}(\tau)$, foi denominada por Koenker e Xiao (2004a) de $\tau$-ésimo quantil de auto-regressão. Dado $\widehat{\alpha}(\tau)$, o $\tau$-ésimo quantil condicional de $y_{t}$, condicional a informações passadas $x_{t}$, pode ser estimado por

$$
\widehat{Q}_{y_{t}}\left(\tau \mid x_{t}\right)=x_{t}^{\top} \widehat{\alpha}(\tau) .
$$

A fim de descrever o resultado central do presente artigo, considere a ilustração fornecida por Koenker e Xiao (2004a) de um processo que apresenta assimetrias ao longo da distribuição condicional. Tome o modelo $Q A R(1)$, em que

$$
Q_{y_{t}}\left(\tau \mid y_{t-1}\right)=\alpha_{0}(\tau)+\alpha_{1}(\tau) y_{t-1},
$$

$\operatorname{com} \alpha_{0}(\tau)=\sigma \Phi^{-1}(\tau)$ e $\alpha_{1}(\tau)=\min \left\{\gamma_{0}+\gamma_{1} \tau, 1\right\}$ para $\gamma_{0} \in(0,1)$ e $\gamma_{1}>0$. Aqui, $\Phi^{-1}(\tau)$ representa a função quantil da distribuição normal padrão, que acumula probabilidade $\tau$, e $\left\{U_{t}\right\}$ é uma seqüência de variáveis aleatórias i.i.d. com distribuição uniforme padrão.

Neste modelo, se $U_{t}>\left(1-\gamma_{0}\right) / \gamma_{1}$, então $y_{t}$ é gerado de acordo com o modelo gaussiano padrão de raiz unitária; porém, para realizações menores de $U_{t}$ tem-se comportamento de reversão à média. Assim, o processo exibe uma forma de persistência assimétrica no sentido que sequiências de inovações fortemente positivas tendem a reforçar o comportamento de raiz unitária, enquanto que realizações de $U_{t}$ inferiores a $\left(1-\gamma_{0}\right) / \gamma_{1}$ induzem reversão à média. Note, todavia, que o processo é globalmente estacionário, ou seja, o comportamento do processo na cauda superior, que é não-estacionário, não domina a dinâmica global da série. Em suma, uma série globalmente estacionária pode apresentar comportamento não-estacionário em certas regiões de sua distribuição condional, tipicamente na cauda superior desta distribuição.

Uma forma alternativa do modelo de auto-regressão quantílica baseia-se na regressão ADF e será apresentada na Seção 4. Esta representação é freqüentemente usada em aplicações econômicas com a finalidade de testar a hipótese de raiz unitária.

\section{TESTES DE RAIZ UNITÁRIA}

A existência de uma raiz unitária em séries temporais surge quando o polinômio auto-regressivo de um modelo ARMA tem uma raiz sobre o círculo unitário. Uma raiz unitária nesse polinômio apresenta considerável interesse em aplicações, devido às suas importantes implicações para a modelagem subjacente. Por exemplo, uma raiz unitária no polinômio auto-regressivo sugere que a série segue um processo não-estacionário integrado de ordem um e, assim, tranformações como diferenças da série podem ser usadas para alcançar estacionariedade. Nesta seção, nós consideramos procedimentos inferenciais para detectar a presença de raiz unitária em polinômios auto-regressivos. Modelos de séries temporais auto-regressivos com uma raiz unitária têm recebido bastante atenção na literatura, especialmente em aplicações econométricas.

A literatura sobre raiz unitária envolvendo dados de taxa de inflação brasileira é volumosa, incluindo os trabalhos de Cati et alii (1999) e de Campêlo e Cribari-Neto (2003), entre outros. Um dos principais interesses no estudo empírico da evolução de séries de taxa de inflação é quanto à estacionariedade das mesmas. Um aspecto associado muito importante, do ponto de vista econômico, é a investigação da hipótese de inflação inercial. Os resultados obtidos variam, naturalmente, de economia para economia, mas dependem também dos métodos estatísticos adotados. Um modelo econométrico para a taxa de inflação do Brasil antes do Plano Real, de acordo com a hipótese de inflação inercial, foi formulado por 
Novaes (1993), que encontrou que a taxa de inflação possui uma raiz unitária. A autora concluiu ainda que cerca de $1 / 3$ da inflação brasileira era de teor inercial. Evidências em favor da hipótese de inflação inercial, através de testes de hipótese de raiz unitária para a taxa de inflação, podem ser encontradas em Cardoso (1983). A existência de uma raiz unitária na taxa de inflação faz com que choques sejam incorporados de forma permanente na estrutura da inflação, impedindo reversão à média. Persistência plena ocorre quando o processo inflacionário, além de conter uma raiz unitária, tem comportamento de passeio aleatório.

Com a implementação do Plano Real, em 1994, acredita-se que foi removido das taxas de inflação do Brasil o componente de retroalimentação inflacionária correspondente a um coeficiente auto-regressivo igual a um (Vieira e Laurini, 2003). Nós realizaremos testes para detectar a presença de raiz unitária em polinômios auto-regressivos para séries de taxas de inflação. Existem vários testes desenvolvidos com este propósito, dentre os quais destacam-se os testes Dickey-Fuller (DF) e Dickey-Fuller aumentado (ADF). Estes testes examinam a hipótese nula de que o processo auto-regressivo possui uma raiz unitária. Aplicaremos às séries da taxa de inflação brasileira o teste Dickey-Fuller aumentado e também testes de raiz unitária baseados em QAR.

O teste $\mathrm{ADF}$ da hipótese nula de uma raiz unitária é realizado a partir da estimação de uma autoregressão de $y_{t}$ em função de $y_{t-1}$ e dos termos defasados de $\Delta y_{t}$ por mínimos quadrados, usando a especificação

$$
y_{t}=\alpha_{0}+\alpha_{1} y_{t-1}+\sum_{j=1}^{k} \alpha_{j+1} \Delta y_{t-j}+u_{t},
$$

conhecida como regressão ADF. Aqui, $\Delta y_{t-j}=y_{t-j}-y_{t-j-1}$, para $j=1, \ldots, k$. Neste modelo, o coeficiente auto-regressivo $\alpha_{1}$ exerce um importante papel na medida de persistência de séries temporais econômicas e financeiras. Se $\alpha_{1}=1$, então $y_{t}$ contém uma raiz unitária e inovações apresentam persistência; por outro lado, se $\left|\alpha_{1}\right|<1$, então $y_{t}$ é integrado de ordem zero. A hipótese nula de uma raiz unitária é formulada como $\mathcal{H}_{0}: \alpha_{1}=1$. Valores críticos para o teste ADF encontram-se tabelados em Fuller (1976, p. 373). Para mais detalhes sobre o teste, ver Said e Dickey (1984). Embora o teste ADF seja útil para testar a hipótese nula de não-estacionariedade, ele pode não ser robusto quando os dados provêm de um processo com distribuição de caudas pesadas e, com isso, pode apresentar baixo poder. Testes alternativos baseados em representações QAR foram recentemente propostos por Koenker e Xiao (2004b). Uma motivação para o uso de testes de raiz unitária baseados em QAR é sua robustez.

Testes de raiz unitária podem ser construídos com base em métodos de auto-regressão quantílica para alguns quantis selecionados da resposta $y_{t}$. Com isso, estes testes proporcionam uma investigação mais detalhada da presença de raiz unitária. Assim, outra motivação para o uso dos testes baseados em QAR, propostos por Koenker e Xiao (2004b), é que uma série temporal pode comportar-se como um processo de raiz unitária (não-estacionário) em certos períodos, mas pode comportar-se como um processo estacionário em outros períodos. Sabe-se que muitas séries econômicas apresentam alguma forma particular de resposta a choques econômicos, embora muitas vezes tal resposta não seja duradoura. Neste sentido, as representações QAR fornecem um enfoque alternativo para testes de raiz unitária. Adicionalmente, métodos de regressão quantílica fornecem técnicas mais robustas para a investigação da presença de uma raiz unitária não apenas para a mediana condicional, mas também para outros quantis do processo.

Nesta seção, baseado no teste proposto por Koenker e Xiao (2004b), consideramos a representação ADF descrita anteriormente, conhecida como regressão ADF. Satisfazendo o princípio da monotonicidade da função quantílica de $y_{t}$, o modelo de regressão ADF pode ser expresso em termos da função quantil condicional de $y_{t}$, em que os coeficientes auto-regressivos são fixos, como

$$
Q_{y_{t}}\left(\tau \mid z_{t}\right)=Q_{u}(\tau)+\alpha_{1} y_{t-1}+\sum_{j=1}^{k} \alpha_{j+1} \Delta y_{t-j} .
$$


No modelo acima, fazendo $\alpha_{0}(\tau)=Q_{u}(\tau), \alpha_{j}(\tau)=\alpha_{j}$, para $j=1, \ldots, k+1$, e definindo $\alpha(\tau)=$ $\left(\alpha_{0}(\tau), \alpha_{1}, \ldots, \alpha_{k+1}\right)$ e $x_{t}=\left(1, y_{t-1}, \Delta y_{t-1}, \ldots, \Delta y_{t-k}\right)^{\top}$, temos que

$$
Q_{y_{t}}\left(\tau \mid z_{t}\right)=x_{t}^{\top} \alpha(\tau)
$$

Em adição ao teste $\mathrm{ADF}$ convencional, nós realizamos testes de raiz unitária baseados em QAR usando a estatística de teste

$$
U_{T}(\tau)=T\left(\widehat{\alpha}_{1}(\tau)-1\right)
$$

em que $T$ denota o número de observações. Para algum quantil $\tau$ fixado, a estatística $U_{T}(\tau)$ é obtida da estimativa do coeficiente $\alpha_{1}(\tau)$ do modelo de regressão ADF fornecida por regressão quantílica. Koenker e Xiao (2004b) estudaram a distribuição limite desta estatística e mostraram que ela é nãopadrão e depende de parâmetros de complicada estimação. Um outro enfoque estudado por eles foi o uso de valores críticos gerados por métodos de reamostragem, ou seja, o uso de pontos críticos obtidos da distribuição nula estimada de $U_{T}(\tau)$. Neste artigo, nós exploramos apenas este último enfoque para testes de raiz unitária baseados em processos QAR. Numa comparação dos procedimentos baseados no modelo QAR, Koenker e Xiao (2004b) mostraram que, em amostras finitas, os testes realizados a partir de valores críticos bootstrap apresentam tamanhos mais próximos aos níveis nominais correspondentes do que testes que usam valores críticos baseados na distribuição nula assintótica. O procedimento utilizado para obtenção dos valores críticos por bootstrap encontra-se descrito em Koenker e Xiao (2004b).

Koenker e Xiao (2004b) propuseram outro método para testar a hipótese de raiz unitária. Eles construíram testes tipo Kolmogorov-Smirnov e Cramer-von-Mises baseados em métodos de regressão quantílica. Nestes testes, em vez de realizar testes locais de raiz unitária, ou seja, examinar a hipótese de raiz unitária apenas em um quantil selecionado por vez, é possível examinar esta hipótese em um grupo de quantis $\tau \in \mathcal{T}=\left[\tau_{0}, 1-\tau_{0}\right]$ para um pequeno $\tau_{0}>0$. Neste artigo, nós utilizamos o teste Kolmogorov-Smirnov proposto por eles para examinar a hipótese de estacionariedade global. Baseada em $U_{T}(\tau)$, a estatística de teste Kolmogorov-Smirnov por regressão quantílica é

$$
Q K S_{\alpha}=\sup _{\tau \in \mathcal{T}}\left|U_{T}(\tau)\right|
$$

A distribuição limite sob a hipótese nula desta estatística de teste pode ser estimada utilizando um esquema de reamostragem de bootstrap.

\section{A DINÂMICA INFLACIONÁRIA BRASILEIRA}

Nós estimamos modelos auto-regressivos tradicionais com $p$ variando de 1 a 12 usando dados da taxa de inflação brasileira, a partir das séries do IGP-DI e do IPCA. O valor ótimo de $p$ para cada série foi selecionado através da minimização do Bayesian Information Criterion (BIC) de Schwarz (1978) e a estimação dos parâmetros auto-regressivos foi realizada por máxima verossimilhança (MV). O modelo selecionado para as séries do IGP-DI e do IPCA foi aquele com $p=1$. Nós investigamos as funções estimadas de autocorrelação residual do modelo AR(1) para ambas as séries. Para a série do IGP-DI, todas as autocorrelações residuais encontraram-se dentro do intervalo assintótico de $95 \%$ de confiança, e para a série do IPCA apenas uma delas estava fora deste intervalo, sugerindo assim que a representação autoregressiva AR(1) é suficiente para filtrar a correlação serial em ambos os casos. Realizamos ainda o teste Ljung-Box da hipótese nula de não-correlação da série residual. Este teste forneceu $p$-valores de 0.9985 e 0.0837 , respectivamente, para as séries residuais IGP-DI e IPCA; assim, não rejeitamos a hipótese de não-correlação da série residual ao nível de significância de 5\%. Desta forma, concluímos que o modelo AR(1) está bem especificado para representar ambas as séries. Os coeficientes auto-regressivos estimados foram 0.612 (IGP-DI) e 0.756 (IPCA).

Em seguida, nós estendemos a análise das dinâmicas destas séries através de modelos de autoregressão quantílica. O modelo QAR(1) foi estimado para 99 quantis condicionais da taxa de inflação 

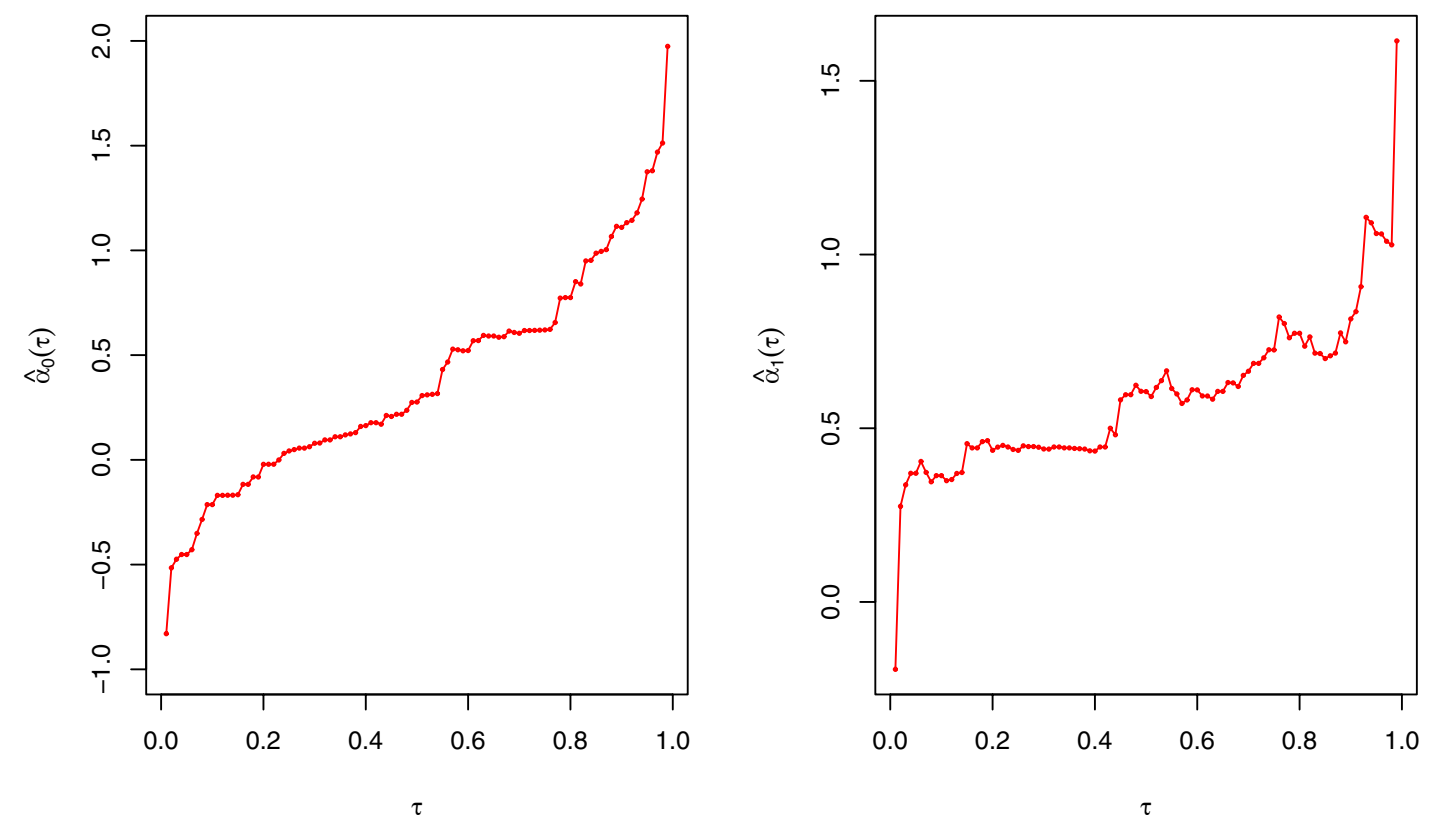

Figura 2 - Coeficientes auto-regressivos quantílicos estimados (IGP-DI).
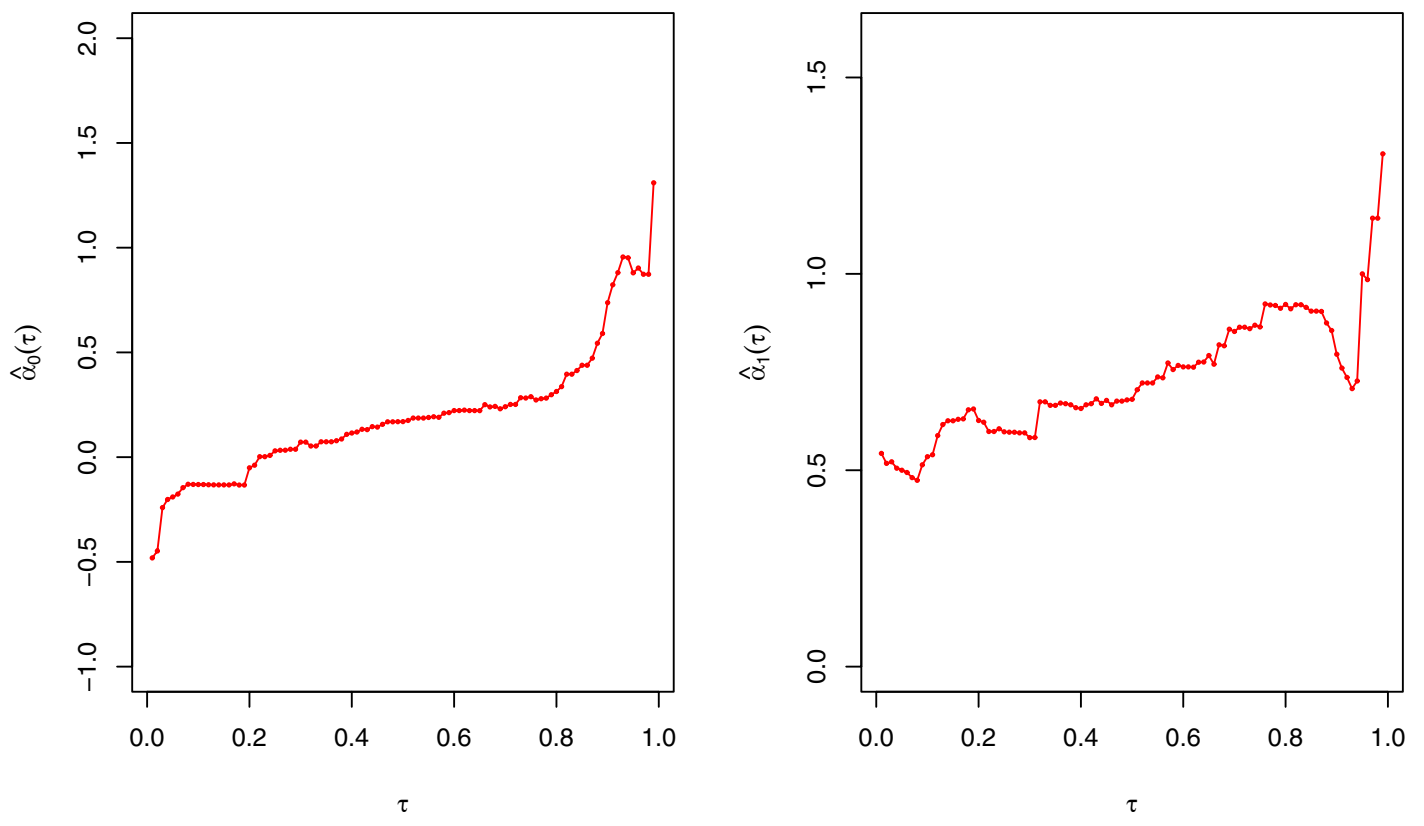

Figura 3 - Coeficientes auto-regressivos quantílicos estimados (IPCA). 
Tabela 1 - Teste de raiz unitária baseado em processo QAR: IGP-DI.

\begin{tabular}{c|c|c|c|c}
\hline Quantil & $\widehat{\alpha}_{1}(\tau)$ & $U_{T}(\tau)$ & \multicolumn{2}{|c}{ Valor crítico } \\
\cline { 3 - 5 } & & & $5 \%$ & $95 \%$ \\
\hline 0.05 & 0.242 & -88.72 & -39.00 & 8.60 \\
0.10 & 0.363 & -74.48 & -28.73 & 2.71 \\
0.15 & 0.303 & -81.52 & -21.65 & 1.99 \\
0.20 & 0.323 & -79.24 & -19.66 & 1.70 \\
0.25 & 0.388 & -71.56 & -18.75 & 1.27 \\
0.30 & 0.413 & -68.63 & -16.91 & 1.35 \\
0.35 & 0.402 & -69.85 & -16.27 & 1.20 \\
0.40 & 0.448 & -64.60 & -14.48 & 1.01 \\
0.45 & 0.507 & -57.69 & -14.31 & 1.12 \\
0.50 & 0.505 & -57.93 & -14.13 & 1.09 \\
0.55 & 0.601 & -46.62 & -14.34 & 1.27 \\
0.60 & 0.543 & -53.46 & -14.34 & 1.31 \\
0.65 & 0.616 & -44.92 & -14.20 & 1.40 \\
0.70 & 0.661 & -39.65 & -15.10 & 1.41 \\
0.75 & 0.741 & -30.28 & -15.88 & 1.66 \\
0.80 & 0.807 & -22.59 & -17.90 & 1.96 \\
0.85 & 0.773 & -26.55 & -19.58 & 2.20 \\
0.90 & 0.781 & -25.58 & -24.51 & 3.95 \\
0.95 & 1.141 & 16.53 & -33.16 & 6.43 \\
\hline
\end{tabular}

Tabela 2 - Teste de raiz unitária baseado em processo QAR: IPCA.

\begin{tabular}{c|c|c|c|c}
\hline Quantil & $\widehat{\alpha}_{1}(\tau)$ & $U_{T}(\tau)$ & \multicolumn{2}{|c}{ Valor crítico } \\
\cline { 3 - 4 } & & & $5 \%$ & $95 \%$ \\
\hline 0.05 & 0.515 & -56.73 & -28.19 & 6.46 \\
0.10 & 0.458 & -63.39 & -21.12 & 3.77 \\
0.15 & 0.424 & -67.43 & -17.57 & 2.33 \\
0.20 & 0.483 & -60.50 & -16.83 & 2.05 \\
0.25 & 0.475 & -61.41 & -16.72 & 1.52 \\
0.30 & 0.560 & -51.53 & -17.25 & 1.51 \\
0.35 & 0.592 & -47.70 & -17.06 & 0.95 \\
0.40 & 0.620 & -44.43 & -15.56 & 1.06 \\
0.45 & 0.639 & -42.27 & -15.13 & 1.24 \\
0.50 & 0.629 & -43.37 & -15.65 & 1.20 \\
0.55 & 0.637 & -42.46 & -16.04 & 1.22 \\
0.60 & 0.717 & -33.14 & -16.32 & 1.24 \\
0.65 & 0.687 & -36.67 & -15.87 & 1.15 \\
0.70 & 0.710 & -33.95 & -16.28 & 1.09 \\
0.75 & 0.714 & -33.48 & -17.82 & 1.35 \\
0.80 & 0.789 & -24.72 & -19.96 & 1.85 \\
0.85 & 0.839 & $-18.84 *$ & -25.82 & 2.74 \\
0.90 & 0.742 & $-30.13 *$ & -32.76 & 4.75 \\
0.95 & 0.591 & -47.83 & -42.79 & 7.21 \\
\hline
\end{tabular}

(*) indica não-rejeição de $\mathcal{H}_{0}: \alpha_{1}=1$. 
igualmente espaçados em $[0.01,0.99]$. Nas Figuras 2 e 3 estão apresentados, de modo evolutivo, os coeficientes auto-regressivos estimados, $\widehat{\alpha}_{0}(\tau)$ e $\widehat{\alpha}_{1}(\tau)$, fornecidos pelo modelo $\operatorname{QAR}(1)$ para as séries do IGP-DI e do IPCA. Para o intercepto, em ambos os casos, nós observamos uma tendência crescente das estimativas com o aumento do quantil condicional. Para as estimativas do coeficiente auto-regressivo $\alpha_{1}(\tau)$, observamos que, para os quantis condicionais mais baixos, os coeficientes auto-regressivos estimados, $\widehat{\alpha}_{1}(\tau)$, apresentam valores baixos, indicando clara tendência de reversão à média. Já para os quantis condicionais mais altos os valores estimados $\widehat{\alpha}_{1}(\tau)$ alcançam e até ultrapassam a unidade, fornecendo indícios de comportamento não-estacionário, explosivo até. Contudo, a maioria dos valores estimados é inferior à unidade, sugerindo estacionariedade global de ambas as séries pós-Plano Real. De modo geral, observamos que a taxa de inflação brasileira tende a apresentar maior grau de persistência nos quantis condicionais mais elevados.

Os resultados apresentados até o momento indicam que a dinâmica inflacionária brasileira apresenta comportamento distinto nos diferentes quantis condicionais, ou seja, seu comportamento depende da localização da taxa de inflação na distribuição condicional. Esta importante característica da dinâmica inflacionária brasileira não foi revelada pelos trabalhos até então publicados.

A investigação da presença de uma raiz unitária em séries de taxa de inflação tem sido objeto de muitos trabalhos econométricos. A seguir, nós examinamos a presença de raiz unitária nas séries da taxa de inflação brasileira medida pelo IGP-DI e pelo IPCA. Primeiro, aplicamos o teste de raiz unitária $\mathrm{ADF}$ convencional a estas séries. Na seleção do número de lags $k$ para ajuste do modelo de regressão ADF foi utilizado o enfoque de minimização do Akaike Information Criterion (AIC). Agiakloglou e Newbold (1996) recomendam o uso do critério AIC para seleção do número de defasagens em testes de raiz unitária $\mathrm{ADF}$. Os modelos de regressão $\mathrm{ADF}$ foram ajustados com $k$ variando de $1 \mathrm{a} 10$. Para as duas séries, a defasagem selecionada para a regressão $\mathrm{ADF}$ foi $k=1$, que forneceu os valores 254.74 (IGPDI) e 121.57 (IPCA) para o AIC. Ao nível de significância de 5\%, o teste ADF tradicional, com $k=1$, rejeita a hipótese de raiz unitária para as duas séries, apresentando $p$-valor menor que 0.0100 para a série do IGP-DI e 0.0109 para a série do IPCA. Estes resultados conduzem à conclusão que o processo inflacionário brasileiro pós-Plano Real não é um processo raiz unitária constante.

Com a finalidade de estudar o comportamento dinâmico da inflação brasileira ao longo de sua distribuição condicional, nós reexaminamos estas duas séries usando o teste de raiz unitária baseado em representações QAR. Primeiro, nós realizamos o teste local para 19 quantis condicionais da taxa de inflação igualmente espaçados em $[0.05,0.95]$. Os valores críticos foram obtidos por bootstrap; o número de réplicas de bootstrap foi $B=1000$. Os resultados estão apresentados nas Tabelas 1 e 2 . A segunda coluna de cada tabela apresenta o coeficiente auto-regressivo estimado, $\widehat{\alpha}_{1}(\tau)$, através da regressão ADF para cada quantil considerado. Através destas estimativas, temos evidências de que, para as duas séries analisadas, existe assimetria no padrão de persistência. De modo geral, nos dois casos, $\widehat{\alpha}_{1}(\tau)$ é incrementado quando passamos dos quantis mais baixos para os quantis mais altos. Esta característica é mais evidente para a série do IGP-DI. Note que são poucos os quantis que apresentaram coeficiente estimado próximo da unidade. Na terceira coluna de cada tabela estão apresentados os valores da estatística $U_{T}(\tau)$. Dada a possibilidade de as séries apresentarem comportamento localmente estacionário ou localmente explosivo, nós realizamos testes contra as hipóteses alternativas de estacionariedade e de explosividade. A quarta e a quinta coluna destas tabelas apresentam os quantis $5 \%$ e $95 \%$ (valores críticos gerados via bootstrap) da distribuição nula de $U_{T}(\tau)$ obtida sob a hipótese nula de raiz unitária usando o procedimento de reamostragem proposto por Koenker e Xiao (2004b).

Através do teste de raiz unitária baseado em representações QAR, para a série do IGP-DI, Tabela 1, a hipótese de raiz unitária é rejeitada, ao nível de significância de 5\%, para todos os quantis condicionais considerados. Porém, vale salientar que no quantil $\tau=0.95$ a hipótese nula é rejeitada contra a hipótese alternativa de raiz explosiva. Assim, concluímos que neste quantil a série do IGP-DI exibe comportamento explosivo. Para a série do IPCA, Tabela 2, a hipótese de raiz unitária não é rejeitada apenas para os quantis 0.85 e 0.90 , sugerindo que esta série exibe comportamento de raiz unitária nos quantis condicionais mais altos. No entanto, para as duas séries da taxa de inflação brasileira, a 
hipótese de raiz unitária é rejeitada na maioria dos quantis considerados. Estas considerações nos levam a concluir que a dinâmica inflacionária brasileira após a implementação do Plano Real não apresenta comportamento uniforme ao longo dos diferentes quantis condicionais do processo inflacionário.

Após realizar o teste local de raiz unitária, nós examinamos a hipótese de raiz unitária para um grupo de quantis através da estatística de teste Kolmogorov-Smirnov, $Q K S_{\alpha}$. Os valores críticos, $C_{K S_{\alpha}}(5 \%)$, obtidos sob a hipótese nula de raiz unitária usando o procedimento de reamostragem proposto por Koenker e Xiao (2004b) para as séries do IGP-DI e do IPCA foram, respectivamente, $11.40 \mathrm{e}$ 10.68. Para ambas as séries, os valores críticos foram inferiores aos da estatística $Q K S_{\alpha}$, respectivamente, 173.80 e 69.05 . Com isso, rejeita-se a hipótese de raiz unitária ao nível de $5 \%$ de significância e, portanto, concluímos que há evidência de que as duas séries analisadas apresentam comportamento globalmente estacionário.

Em particular, com base nos resultados obtidos, concluímos que a dinâmica inflacionária brasileira aparenta ser globalmente estacionária, apesar de haver distanciamento da estacionariedade na cauda superior da distribuição condicional. Este comportamento assimétrico não foi identificado por estudos anteriores.

\section{CONCLUSÃO}

Neste artigo nós estudamos a dinâmica inflacionária brasileira pós-Plano Real ao longo da distribuição condicional da taxa de inflação, examinando a existência de raiz unitária, ou seja, a existência de inércia inflacionária. Usamos a classe de modelos auto-regressivos quantílicos com a finalidade de investigar assimetrias na dinâmica inflacionária brasileira. Os modelos QAR possuem vantagens sobre os modelos clássicos baseados em mínimos quadrados no estudo de séries temporais provenientes de distribuições de caudas pesadas. Em adição, estes modelos fornecem um método mais robusto de estimação, propriedade muito importante na modelagem de séries que apresentam observações extremas, como é o caso da taxa de inflação brasileira.

Nós mostramos que as duas séries da taxa mensal de inflação brasileira, IGP-DI e IPCA, são adequadamente representadas pelo modelo auto-regressivo quantílico mais simples, QAR(1). Como resultado principal, obtivemos que estas séries exibem dinâmicas assimétricas. Os coeficientes auto-regressivos estimados $\widehat{\alpha}_{1}(\tau)$ assumem diferentes valores ao longo da distribuição condicional da taxa de inflação. Em particular, os valores de $\widehat{\alpha}_{1}(\tau)$ nos quantis inferiores são menores que os valores de $\widehat{\alpha}_{1}(\tau)$ nos quantis superiores, indicando que o comportamento local da taxa de inflação brasileira durante períodos de baixa inflação apresenta menor sensibilidade a choques.

Nós aplicamos também um teste de raiz unitária baseado em representações QAR às séries da taxa de inflação brasileira. Muitos estudos empíricos têm investigado a existência de raiz unitária em séries de taxa de inflação, o que indica existência de inércia inflacionária. Os resultados a respeito de tal inércia no processo inflacionário brasileiro são distintos. Novaes (1993) estimou o grau de inércia na inflação brasileira em 1/3. Durevall (1998) estimou o nível de inflação inercial no Brasil em 0.41. Cati et alii (1999) encontraram que a dinâmica inflacionária brasileira é quase inteiramente inercial, 0.97 . Em contraste, Campêlo e Cribari-Neto (2003) encontraram que a inércia na dinâmica inflacionária brasileira é negligível.

Antes de aplicarmos os testes de raiz unitária baseados em QAR, nós aplicamos o teste ADF convencional. Através deste teste nós concluímos que a série da taxa de inflação do Brasil após a implementação do Plano Real não exibe raiz unitária, indicando que o processo inflacionário não é um processo raiz unitária constante. Os testes baseados em representações QAR fornecem uma descrição mais detalhada da série, permitindo a avaliação da propriedade de raiz unitária em vários quantis da distribuição condicional. As evidências baseadas nas estimativas do coeficiente auto-regressivo $\widehat{\alpha}_{1}(\tau)$ em cada quantil sugerem que o processo inflacionário do Brasil, de fato, não é um processo raiz unitária constante. Em 
particular, os resultados evidenciam que a dinâmica inflacionária brasileira é globalmente estacionária, mesmo o processo alcançando não-estacionariedade na cauda superior da distribuição condicional.

Por fim, nossos resultados indicam que o comportamento dinâmico do processo inflacionário brasileiro não é constante ao longo da distribuição condicional da taxa de inflação. A dinâmica inflacionária brasileira comporta-se exatamente como a ilustração fornecida por Koenker e Xiao (2004a) de um processo que apresenta assimetrias ao longo da distribuição condicional: estacionariedade global, mas não-estacionariedade na cauda superior da distribuição condicional. Em particular, choques ocorridos quando o processo se encontra na cauda superior de tal distribuição, ou seja, em períodos de inflação elevada, possuem tempo de dissipação superior àqueles choques que ocorrem quando o processo está em outras regiões da distribuição condicional. Dado o caráter globalmente estacionário da série, todas as inovações possuem efeitos apenas de curto prazo, mas o tempo de dissipação de movimentos inesperados parece depender do comportamento local da dinâmica inflacionária. Essa dinâmica assimétrica não havia sido revelada por trabalhos anteriores.

\section{Referências Bibliográficas}

Agiakloglou, C. \& Newbold, P. (1996). The balance between size and power in Dickey-Fuller tests with data-dependent rules for the choice of truncation lag. Economics Letters, 52(3):229-234.

Ball, L., Mankiw, N., \& Page, M. (1992). Asymmetric Price Adjustment and Economic Fluctuations. NBER. Working Paper 4089.

Campêlo, A. \& Cribari-Neto, F. (2003). Inflation inertia and inliers: the case of Brazil. Revista Brasileira de Economia, 57:713-719.

Cardoso, E. (1983). Indexação e acomodação monetária: um teste do processo inflacionário brasileiro. Revista Brasileira de Economia, 31(1):3-11.

Cassiano, K. (2003). Uma análise da dinâmica inflacionária brasileira. Dissertação de mestrado, Departamento de Estatística, Universidade Federal de Pernambuco.

Cati, R., Garcia, M., \& Perron, P. (1999). Unit roots in the presence of abrupt governmental interventions with an application to Brazilian data. Journal of Applied Econometrics, 14(1):27-56.

Durevall, D. (1998). The Dynamics of Chronic Inflation in Brazil, 1968-1985. Journal of Business \& Economic Statistics, 16(4):423-432.

Fuller, W. (1976). Introduction to statistical time series. Wiley, New York.

Hasan, M. \& Koenker, R. (1997). Robust rank tests of the unit root hypothesis. Econometrica, 65(1):133161.

Knight, K. (1989). Limit theory for autoregressive-parameter estimates in an infinite-variance random walk. Canadian Journal of Statistics, 17(3):261-278.

Koenker, R. \& Bassett Jr, G. (1978). Regression quantiles. Econometrica, 46(1):33-50.

Koenker, R. \& Xiao, Z. (2004a). Quantile autoregression. Texto para discussão, Disponível em: http: //www. econ.uiuc.edu/ roger/research/qar/qar9.pdf.

Koenker, R. \& Xiao, Z. (2004b). Unit root quantile autoregression inference. Journal of the American Statistical Association, 99(467):775-787. 
Novaes, A. (1993). Revisiting the inertial inflation hypothesis for Brazil. Journal of Development Economics, 42:89-110.

Rogers, A. (2001). Least absolute deviations regression under nonstandard conditions. Econometric Theory, 17(4):820-852.

Said, S. \& Dickey, D. (1984). Testing for unit roots in autoregressive-moving average models of unknown order. Biometrika, 71:599-608.

Schwarz, G. (1978). Estimating the dimension of a model. The Annals of Statistics, 6(2):461-464.

Vieira, H. \& Laurini, M. (2003). Inércia nas taxas de inflação: um modelo heterocedástico em espaço de estado. Texto para discussão.

Wallis, K. (1999). Asymmetric density forecasts of inflation and the Bank of England's fan chart. National Institute Economic Review, 167:106-113.

Weiss, A. (1991). Estimating nonlinear dynamic models using least absolute error estimation. Econometric Theory, 7:46-68. 